\title{
COVID-19 and eosinophilic granulomatosis with polyangiitis or COVID-19 mimicking eosinophilic granulomatosis with polyangiitis?
}

\author{
Bahar Özdemir ${ }^{1}$ (1) $\cdot$ Abdulsamet Erden ${ }^{1} \cdot$ Serdar Can Güven $^{1} \cdot$ Berkan Armagan ${ }^{1} \cdot$ Hakan Apaydin $^{1} \cdot$ Özlem Karakas $^{1}$. \\ Ahmet Gokhan Akdag ${ }^{2}$ ìhsan Ates ${ }^{3}$. Orhan Kucuksahin ${ }^{4} \cdot$ Ahmet Omma $^{1}$
}

Received: 23 March 2021 / Accepted: 15 May 2021

(c) The Author(s), under exclusive licence to Springer-Verlag GmbH Germany, part of Springer Nature 2021, corrected publication 2021

\begin{abstract}
Coronavirus disease 2019 (COVID-19) and eosinophilic granulomatosis with polyangiitis (EGPA) share similarities in clinical, imaging findings and may present with respiratory distress. Differentiating a new-onset EGPA from COVID-19 during the current pandemic is a diagnostic challenge, particularly if other EGPA symptoms are overlooked. Here in this study we reviewed the literature regarding EGPA patients with COVID-19 and patients who diagnosed with EGPA or suffered an EGPA flare mimicking COVID-19. We conducted a literature survey in PUBMED database using meshed keywords "COVID-19" and "EGPA", "COVID-19" and "eosinophilic granulomatosis with polyangiitis", "COVID-19" and "Churg Strauss Syndrome", to reveal previously reported cases involving EGPA patients who had COVID-19 infection, patients who suspected to have COVID-19 but eventually diagnosed with EGPA and patients with a known diagnosis of EGPA who suffered a flare but a COVID-19 infection was suspected initially. A total of 11 cases ( 6 literature cases, 5 cases from our clinic) were included in our study. Seven (63.6\%) of the cases were defined as COVID-19 mimicker and 4 (36.4\%) were EGPA with COVID-19. All of the cases in EGPA with COVID-19 group had a history of asthma. All of them had a positive PCR result and ground-glass opacities in thorax CT. In COVID-19 mimicker group, six (85.7\%) patients had a history of asthma and other EGPA features that were observed were eosinophilia in 6 (85.7\%). Our study provided clues regarding the EGPA/ COVID-19 diagnostic challenge which may be useful in the current pandemic. Since none of the findings in COVID-19 are disease-specific, other conditions like EGPA should not be overlooked particularly in PCR negative patients and clinical, laboratory and imaging findings should be interpreted carefully. Furthermore, we did not observe poor outcomes in EGPA patients who had COVID-19.
\end{abstract}

Keywords Coronavirus disease 2019 - Severe acute respiratory syndrome—coronavirus $2 \cdot$ Eosinophilic granulomatosis with polyangiitis $\cdot$ Churg strauss syndrome

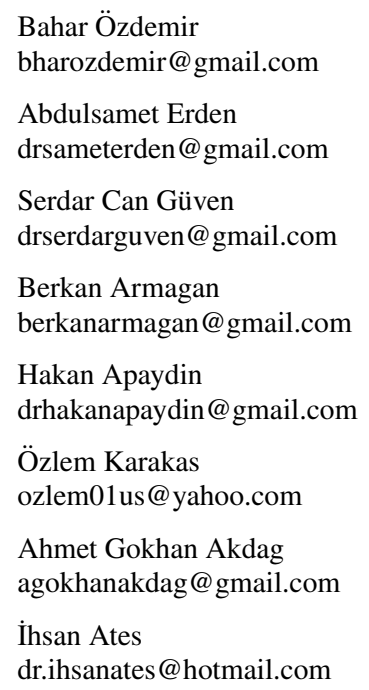

Orhan Kucuksahin orhankcs@gmail.com

Ahmet Omma

ahmetomma@gmail.com

1 Division of Rheumatology, Department of Internal Medicine, Ankara City Hospital, Ankara 06100, Turkey

2 Division of Intensive Care, Department of Internal Medicine, Ankara City Hospital, Ankara, Turkey

3 Department of Internal Medicine, Ankara City Hospital, Ankara, Turkey

4 Division of Rheumatology, Department of Internal Medicine, Ankara City Hospital, Yıldırım Beyazıt University, Ankara, Turkey 


\section{Introduction}

Pandemic of coronavirus disease 2019 (COVID-19), caused by severe acute respiratory syndrome-coronavirus 2 (SARS-CoV 2), has become a major global health issue since December 2019. Although most patients suffer mildly a considerable number of patients, particularly with older age and comorbidities, have a severe disease with increased risk of mortality and morbidity. The diagnosis of COVID-19 is generally established with a positive SARS-CoV 2 polymerized chain reaction (PCR) test or COVID-19 antibody test in presence of a constellation of compatible clinical and imaging findings [1]. However, the majority of symptoms and laboratory findings of COVID-19 are usually non-specific, particularly in the early disease course. Symptoms like fever, dyspnea, cough, malaise, myalgia and arthralgia are common in the early stages in addition to more specific symptoms like anosmia and ageusia. Although increased acute phase reactants including C-reactive protein (CRP), erythrocyte sedimentation rate (ESR), ferritin and lymphopenia are suggestive of a COVID-19 infection in presence of meaningful clinical findings, these laboratory changes can accompany various other infectious and inflammatory conditions [2]. Likewise, common imaging findings of COVID-19 such as multifocal, patchy ground-glass opacities and consolidations in thorax computed tomography (CT) can also be observed in many other conditions [3]. Therefore, especially in absence of PCR or serological findings of COVID-19, differential diagnosis is wide, comprising conditions like other infectious pneumonia, drug-induced lung toxicity, interstitial lung disease and rheumatic conditions involving lungs such as eosinophilic granulomatosis with polyangiitis (EGPA) $[4,5]$.

Eosinophilic granulomatosis with polyangiitis (EGPA) is an antineutrophil cytoplasmic antibody (ANCA) associated vasculitis characterized by necrotizing and eosinophil rich inflammation involving multiple organ systems including lungs, heart, kidneys, upper respiratory tract, gastrointestinal tract and peripheral nervous system. Lung involvement is one of the cardinal manifestations of EGPA and typically presents with multifocal, peripheral, patchy and migratory parenchymal infiltrates [6]. Accordingly, pulmonary symptoms such as cough and dyspnea accompanied by constitutional symptoms such as fever, malaise, arthralgia and myalgia may be an initial presentation in EGPA. Since both conditions share similarities in clinical, imaging findings and present with respiratory distress, differentiating a new-onset EGPA from COVID-19 during the current pandemic is a diagnostic challenge, particularly if other EGPA symptoms are overlooked. Likewise, an EGPA flare in patients with a prior diagnosis can be misjudged as a COVID-19 infection. Furthermore, there is a lack of knowledge in the literature regarding COVID-19 course and outcomes in EGPA patients.

Here in this study, we reviewed the literature regarding EGPA patients with COVID-19 and patients who diagnosed with EGPA or suffered an EGPA flare mimicking COVID19. We also evaluated similar patients of our clinic and described clinical features of such patients combining our cases and literature cases. Our aim was to determine clinical features of such cases to provide knowledge for clinicians which may be helpful in the differential diagnosis of these entities. Furthermore, we also aimed to elucidate COVID-19 outcomes in EGPA patients.

\section{Materials and method}

We conducted a literature survey in PUBMED database using meshed keywords "COVID-19" and "EGPA", "COVID-19" and "eosinophilic granulomatosis with polyangiitis", "COVID-19" and "Churg Strauss Syndrome", to reveal previously reported cases involving EGPA patients who had COVID-19 infection, patients who suspected to have COVID-19 but eventually were diagnosed with EGPA and patients with a known diagnosis of EGPA who suffered a flare but a COVID-19 infection was suspected initially. Publications in the English language and comprising human subjects were included. After the initial survey, twelve publications potentially related to our research were detected. Abstracts of these 12 publications were evaluated and 5 articles which reported related cases were included in our literature review (Fig. 1). Full texts of these 5 articles were viewed and a total of 6 cases with the clinical features regarding our research topic were detected. In addition to these 6 literature cases we added 5 other cases with similar properties which have been followed in our clinic between March and December 2020. These 5 cases fulfilled the American College of Rheumatology (ACR) 1990 classification criteria for EGPA [7].

Among these 11 cases, patients with suspected COVID19 who ended up with a diagnosis of new-onset EGPA or EGPA flare with a negative COVID-19 PCR test were defined as "COVID-19 mimicker" group. The patients with a prior diagnosis of EGPA and suffered from COVID-19 confirmed by a positive PCR test were defined as "EGPA with COVID-19" group.

Data regarding demographics, comorbidities, clinical, laboratory and imaging findings, medical treatments for EGPA and COVID-19, rates of need for oxygen support and mortality during disease course were recorded in all cases. Official permission was obtained from the Ministry of Health to conduct the study. The study was approved by the 
Fig. 1 Flow chart demonstrating the article evaluation process for recruitment

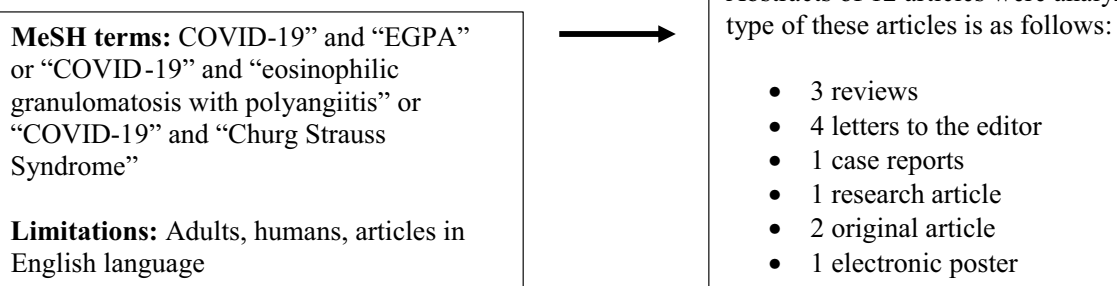

MeSH terms: COVID-19" and "EGPA" or "COVID-19" and "eosinophilic granulomatosis with polyangiitis" or "COVID-19" and "Churg Strauss Syndrome"

Limitations: Adults, humans, articles in English language type of these articles is as follows:

- 3 reviews

- 4 letters to the editor

- 1 case reports

- 1 research article

- 2 original article

- 1 electronic poster

Based on these criteria 7 articles were excluded from the study. The type of excluded articles is as follows:

- 3 reviews

- 1 letter to the editor

- 1 research article

- 2 original article
Abstracts of 12 articles were analyzed. The
5 articles were included in the study.

The type of the articles included in the study is as follows:

- 3 letters to the editor

- 1 case report

- 1 electronic poster
Ethics Committee of Ankara City Hospital with the approval Number E1-21-1692.

Data were analyzed using Statistical Package for the Social Sciences (SPSS) 22.0 software. Shapiro-Wilk's test was used to determine the distribution of the data. The distribution of continuous data was expressed as mean \pm standard deviation. Continuous without normal distribution were expressed as median and minimum-maximum values. For categorical variables, the outcomes were expressed as number and percentages.

\section{Results}

A total of 11 cases ( 6 literature cases, 5 cases from our clinic) were included in our study. Seven $(63.6 \%)$ of the cases were defined as COVID-19 mimicker and 4 (36.4\%) were EGPA with COVID-19.

Median (min-max) age in EGPA with COVID-19 group was 36.5 (25-59) and a single patient was male. None of these patients had a history smoking or alcohol consumption. All of the cases in EGPA with COVID-19 group had a history of asthma and one case additionally had chronic hepatitis B infection. Most common COVID-19 symptoms in this group were fever in $2(50 \%)$, cough in 2 (50\%), malaise in $2(50 \%)$ and myalgia in $2(50 \%)$ cases. All of them had a positive PCR result and ground-glass opacities in thorax CTs. A single patient had eosinophilia (25\%) while $2(50 \%)$ had lymphopenia in peripheral blood. Three $(75 \%)$ had elevated CRP levels. Two (50\%) patients were under low-dose steroids, 2 (50\%) under rituximab and $1(25 \%)$ under cyclophosphamide when diagnosed with COVID-19. During COVID-19 course, 2 (50\%) patients needed oxygen support and no deaths were observed. Demographics and clinical features of EGPA with COVID-19 group were presented in Table 1.

In COVID-19 mimicker group, there were 7 patients (6 with new-onset EGPA and 1 with EGPA flare) with a median (min-max) age of 40 (20-61). Two (28.5\%) of the patients were male. Six $(85.7 \%)$ patients had a history of asthma and other EGPA features that were observed were eosinophilia in $6(85.7 \%)$, history of allergic rhinitis in $2(28.5 \%)$, cardiac 
Table 1 Demographics and clinical features of EGPA patients with COVID-19

\begin{tabular}{llll}
\hline & Literature cases $(n=2)$ & Our cases $(n=2)$ & $\begin{array}{l}\text { Total } \\
(n=4)\end{array}$ \\
\hline Age, median (min-max) & $42(25-59)$ & $36.5(33-40)$ & $36.50(25-59)$ \\
Male, $n(\%)$ & $1(50)$ & 0 & $1(25)$ \\
Asthma, $n(\%)$ & $2(100)$ & $2(100)$ & $4(100)$ \\
COVID-19 symptoms, $n(\%)$ & & & \\
Fever & $1(50)$ & $1(50)$ & $2(50)$ \\
Cough & $1(50)$ & $1(50)$ & $2(50)$ \\
Malaise & $1(50)$ & $1(50)$ & $2(50)$ \\
Myalgia & $1(50)$ & $1(50)$ & $2(50)$ \\
Dyspnea & $0(0)$ & $1(50)$ & $1(25)$ \\
Headache & $1(50)$ & 0 & $1(25)$ \\
Nausea & 0 & $1(50)$ & $1(25)$ \\
Vomiting & 0 & $1(50)$ & $1(25)$ \\
Positive COVID-19 PCR test, $n(\%)$ & $2(100)$ & $2(100)$ & $4(100)$ \\
Eosinophilia, $n(\%)$ & $1(50)$ & 0 & $1(25)$ \\
Lymphopenia, $n(\%)^{\mathrm{a}}$ & - & $2(100)$ & $2(50)$ \\
Ground-glass opacities in thorax CT, $n(\%)$ & $2(100)$ & $2(100)$ & $4(100)$ \\
Elevated CRP $(>5$ mg/l), $n(\%)$ & $1(50)$ & $2(100)$ & $3(75)$ \\
EGPA treatment, $n(\%)$ & $1(50)$ & $1(50)$ & $2(50)$ \\
Low-dose glucocorticoids ${ }^{\mathrm{b}}$ & $1(50)$ & $1(50)$ & $2(50)$ \\
Rituximab & $1(50)$ & 0 & $1(25)$ \\
Cyclophosphomide & $1(50)$ & $1(50)$ & $2(50)$ \\
Need for oxygen support, $n(\%)$ & 0 & 0 & \\
Mortality, $n(\%)$ & & & \\
\hline & & & \\
\hline
\end{tabular}

EGPA eosinophilic granulomatosis with polyangitis, COVID-19 coronavirus disease $2019, n$ number, $\min$ minimum, $\max$ maximum, $P C R$ polymerized chain reaction, $C T$ computed tomography, $C R P$ c-reactive protein

${ }^{a}$ literature cases were missing lymphocyte count

${ }^{b}$ glucocorticoid dose $<10 \mathrm{mg}$ prednisolone equivalent involvement (coronary vasculitis) in $3(42.8 \%)$, hematuria in 2 (28.5\%) history of nasal polyps in $1(14.2 \%)$, myeloperoxidase (MPO) ANCA positivity in 1 (14.2\%), cutaneous eosinophilic infiltrates presenting as polycyclic erythema in $1(14.2 \%)$ and drop-foot in $1(14.2 \%)$ of the patients. Dyspnea was the most common symptom on admission $(n=6,85.7 \%)$, followed by cough $(n=5,71.4 \%)$. All of them had a negative PCR result and ground-glass opacities were present in thorax CTs of all cases. Eosinophilia was absent only in one case which already had an EGPA diagnosis, was under low dose steroids and accepted as an EGPA flare. Four (57.1\%) of the COVID-19 mimickers needed oxygen support and one death was observed $(14.2 \%)$ in this group. Demographics and clinical features of the COVID-19 mimicker group were presented in Table 2.

\section{Discussion}

EGPA is a rheumatic condition with frequent lung involvement characterized by patchy parenchymal infiltrates. Such presentation may create a diagnostic challenge during the pandemic since similar lesions are frequently observed in COVID-19 pneumonia. Our aim was to call attention to this issue and define clinical features which may be helpful in the differential of these entities. Additionally, we investigated COVID-19 outcomes in EGPA patients.

There is no specific symptom defined for COVID-19 yet [2]. COVID-19 is generally diagnosed with a positive PCR and/or coherent imaging findings in patients with symptoms suggestive of the disease [8]. Sensitivity of thorax CT in diagnosis was reported to be superior to PCR (97.2\% vs $83.3 \%$ ) and imaging findings emerge especially in the diagnosis of PCR negative patients [8,9]. Most common imaging findings were revealed to be ground-glass opacities (GGO) (83\%), GGO with consolidations, pleural thickening, interlobular septal thickening and air bronchograms [10]. However, none of these findings are specific to COVID-19 and frequently observed in other infections, interstitial lung diseases and lung involvements of rheumatic conditions such as EGPA. Accordingly, in a study, among 40 PCR negative subjects with suspected COVID-19 infection who eventually diagnosed with other conditions, a 36-year-old female 
Table 2 Demographics and clinical features of COVID-19 mimicker group

\begin{tabular}{|c|c|c|c|}
\hline & Literature cases $(n=4)$ & Our cases $(n=3)$ & $\begin{array}{l}\text { Total } \\
(n=7)\end{array}$ \\
\hline Age, median (min-max) & $35(20-41)$ & $46(30-61)$ & $40(20-61)$ \\
\hline Male, $n(\%)$ & $1(25)$ & $1(33.3)$ & $2(28.5)$ \\
\hline \multicolumn{4}{|l|}{ EGPA features, $n(\%)$} \\
\hline Asthma & $3(75)$ & $3(100)$ & $6(85.7)$ \\
\hline Eosinophilia & $4(100)$ & $2(66.6)$ & $6(85.7)$ \\
\hline Coronary vasculitis & $1(25)$ & $2(66.6)$ & $3(42.8)$ \\
\hline Allergic rhinitis & 0 & $2(66.6)$ & $2(28,5)$ \\
\hline Hematuria & $1(25)$ & $1(33.3)$ & $2(28,5)$ \\
\hline Nasal polyps & $1(25)$ & 0 & $1(14.2)$ \\
\hline MPO-ANCA positivity & $1(25)$ & 0 & $1(14.2)$ \\
\hline Cutaneous lesions & $1(25)$ & 0 & $1(14.2)$ \\
\hline Drop-foot & $1(25)$ & 0 & $1(14.2)$ \\
\hline PR3-ANCA positivity & 0 & 0 & $0(0)$ \\
\hline Proteinuria & 0 & 0 & $0(0)$ \\
\hline Elevated serum creatinine & 0 & 0 & $0(0)$ \\
\hline \multicolumn{4}{|l|}{ Comorbidities, $n(\%)$} \\
\hline Diabetes mellitus & 0 & $1(33.3)$ & $1(14.2)$ \\
\hline Hypertension & 0 & $1(33.3)$ & $1(14.2)$ \\
\hline \multicolumn{4}{|l|}{ Symptoms on admission, $n(\%)$} \\
\hline Dyspnea & $3(75)$ & $3(100)$ & $6(85.7)$ \\
\hline Cough & $2(50)$ & $3(100)$ & $5(71.4)$ \\
\hline Malaise & 0 & $2(66.6)$ & $2(28,5)$ \\
\hline Chest pain & $1(25)$ & $1(33.3)$ & $2(28,5)$ \\
\hline Fever & $1(25)$ & 0 & $1(14.2)$ \\
\hline Myalgia & 0 & $1(33.3)$ & $1(14.2)$ \\
\hline Nausea & 0 & $1(33.3)$ & $1(14.2)$ \\
\hline Vomiting & 0 & $1(33.3)$ & $1(14.2)$ \\
\hline Positive COVID-19 PCR test, $n(\%)$ & 0 & 0 & 0 \\
\hline Eosinophilia, $n(\%)$ & $4(100)$ & $2(66.6)$ & $6(85.7)$ \\
\hline Lymphopenia, $n(\%)^{\mathrm{a}}$ & - & 0 & 0 \\
\hline Ground-glass opacities in thorax CT, $n(\%)$ & $4(100)$ & $3(100)$ & $7(100)$ \\
\hline Elevated CRP (> $5 \mathrm{mg} / \mathrm{l}), n(\%)$ & $3(75)$ & $3(100)$ & $6(85.7)$ \\
\hline Need for oxygen support, $n(\%)$ & $2(50)$ & $2(66.6)$ & $4(57.1)$ \\
\hline Mortality, $n(\%)$ & 0 & $1(33.3)$ & $1(14.2)$ \\
\hline
\end{tabular}

COVID-19 coronavirus disease 2019, $n$ number, min minimum, max maximum, EGPA eosinophilic granulomatosis with polyangitis, $P C R$ polymerized chain reaction, $C T$ computed tomography, $C R P$ c-reactive protein

${ }^{\mathrm{a}}$ literature cases were missing lymphocyte count patient with a history of asthma having multiple GGO with consolidations and crazy-paving pattern in thorax CT was diagnosed with EGPA. EGPA diagnosis was reported to be confirmed with paranasal involvement in CT, arthritis, eosinophilia and elevated Ig E levels [11].

The COVID-19 mimicker group in our study involved 7 patients (6 with new onset EGPA and 1 with EGPA flare). All of the patients were observed to present with respiratory symptoms and COVID-19 was the preliminary diagnosis in all. GGO were present in all cases, however, none of them was PCR positive which was probably the main reason for consideration of other conditions [12-14]. Six of these cases had peripheral eosinophilia as a common feature contradictory to the fact that eosinopenia is more common in COVID19 , reaching a rate of $95 \%$ in some studies [15-18]. On the other hand, eosinophilia is a diagnostic criterion in EGPA and may present in $90 \%$ of cases $[19,20]$. The only patient in our study without eosinophilia already had an EGPA diagnosis and was under steroid treatment which is known to suppress peripheral eosinophilia. Another common feature in our COVID-19 mimicker group was a history of asthma which is again a cardinal feature of EGPA. Other EGPA 
related findings in our study group were a history of allergic rhinitis and nasal polyps, coronary vasculitis, cutaneous eosinophilic erythema, drop-foot (which may be indicative of peripheral neuropathy) and hematuria (which may be indicative of renal involvement). These findings suggest not to overlook EGPA during the pandemic, particularly PCR negative patients should be evaluated thoroughly for accompanying symptoms.

ANCA positivity is generally useful in distinguishing ANCA-associated vasculitides (AAV) from other conditions with similar features. However, the rate of ANCA positivity is relatively low in EGPA when compared to other AAV and reported to vary from 38 to $60 \%$ [21-23]. In contrast to the vasculitic phenotype of EGPA with prominent manifestations of small-vessel vasculitis, rate of ANCA positivity is further reduced in the eosinophilic phenotype of EGPA which is characterized by eosinophilic infiltrates in organ systems [24]. Likewise, in our study ANCAs were positive in only one case (MPO-ANCA). Therefore, ANCA negativity may not be reliable in distinguishing COVID-19 from EGPA in suspected patients.

There were four EGPA patients with PCR confirmed COVID-19 in our study [25, 26]. COVID-19 related mortality was not observed in any of the patients. Similarly, COVID-19 Global Rheumatology Alliance physicianreported registry have already reported no significant increase in COVID-19 related mortality among vasculitis patients [27]. Laboratory data was available in two of the cases, neither of them had eosinophilia at the time of COVID-19 but both of them had lymphopenia. Absence of eosinophilia, presence of lymphopenia and/or eosinopenia in peripheral blood may be in favor of COVID-19 infection rather than an EGPA flare in such cases [15-17, 28-30].

Systemic glucocorticoids are proven to have beneficial effects on COVID-19 outcomes [31]. However, being under systemic glucocorticoid treatment with a dose $\geq 10 \mathrm{mg}$ prednisolone equivalent at the onset of COVID-19 infection has been reported to be related to an increased risk of hospitalization [27]. In our study, 2 of the EGPA patients were under low-dose glucocorticoid treatment (4 mg methylprednisolone in both cases) and despite both of them observed to be hospitalized neither of them needed oxygen support. Rituximab therapy have also been related with poor prognosis in rheumatic patients with COVID-19 infection [27]. As a potent inhibitor of CD20 B lymphocytes, rituximab hampers antibody production and hence the humoral immune system which plays a key role against viral infections [32]. In a multicenter study on AAV patients, 7 deaths were reported in 58 AAV patients with COVID-19 and 4 of those 7 were under rituximab treatment [33]. In our study, 2 EGPA patients were under rituximab treatment at the time of COVID-19 and despite both of them revealed to be hospitalized and had oxygen support, neither of them died.
In the pandemic, in most patients who present with respiratory distress, COVID-19 infection is inevitably the preliminary diagnosis. Since none of the findings in COVID19 are disease-specific, other conditions like EGPA should not be overlooked particularly in PCR negative patients and clinical, laboratory and imaging findings should be interpreted carefully. Small sample size was the major limitation of this study. Secondly, some clinical and laboratory parameters were missing in the literature cases. It is known that nasopharyngeal swab may be false negative in some COVID-19 patients. Sampling with invasive techniques such as bronchofiberoscopy may increase the sensitivity of PCR test. Lack of further sampling with bronchofiberoscopy in PCR negative patients was another limitation of our study. Nevertheless, our study provides clues regarding the EGPA / COVID-19 diagnostic challenge which may be useful in the current pandemic. Furthermore, we did not observe poor outcomes in EGPA patients who had COVID-19. Larger studies will provide further knowledge regarding the management of EGPA during the COVID-19 pandemic.

Funding The author(s) received no financial support for the research, authorship, and/or publication of this article.

\section{Declarations}

Conflict of interest The author(s) declared no potential conflicts of interest with respect to the research, authorship, and/or publication of this article.

\section{References}

1. Chu DK, Pan Y, Cheng SM et al (2020) Molecular diagnosis of a novel coronavirus (2019-nCoV) causing an outbreak of pneumonia. Clin Chem 66(4):549-555

2. Struyf T, Deeks JJ, Dinnes J et al (2020) Signs and symptoms to determine if a patient presenting in primary care or hospital outpatient settings has COVID-19 disease. Cochrane Database Syst Rev. https://doi.org/10.1002/14651858.CD013665

3. Li Y, Xia L (2020) Coronavirus disease 2019 (COVID-19): role of chest CT in diagnosis and management. Am J Roentgenol 214(6):1280-1286

4. Bernheim A, Mei X, Huang M et al (2020) Chest CT findings in coronavirus disease-19 (COVID-19): relationship to duration of infection. Radiology 295(3):200463. https://doi.org/10.1148/ radiol.2020200463

5. Cleverley JR, Screaton NJ, Hiorns MP, Flint JD, Müller NL (2002) Drug-induced lung disease: high-resolution CT and histological findings. Clin Radiol 57(4):292-299

6. Churg J, Strauss L (1951) Allergic granulomatosis, allergic angiitis, and periarteritis nodosa. Am J Pathol 27(2):277

7. Masi AT, Hunder GG, Lie JT et al (1990) The American College of Rheumatology 1990 criteria for the classification of ChurgStrauss syndrome (allergic granulomatosis and angiitis). Arthritis Rheumatol 33(8):1094-1100

8. Patel A, Jernigan DB (2020) Initial public health response and interim clinical guidance for the 2019 novel coronavirus 
outbreak-United States, December 31, 2019-February 4, 2020. Morb Mortal Wkly Rep 69(5):140

9. Long C, Xu H, Shen Q et al (2020) Diagnosis of the coronavirus disease (COVID-19): rRT-PCR or CT? Eur J Radiol 126:108961

10. Bao C, Liu X, Zhang H, Li Y, Liu J (2020) Coronavirus disease 2019 (COVID-19) CT findings: a systematic review and metaanalysis. J Am Coll Radiol 17(6):701-709

11. Motawea AM, Omar S, Yasin R (2021) Imaging of COVID-19 simulators. Egypt J Radiol Nucl Med 52(1):1-17

12. Tan Y, Mohamedalhadi A, Wood F (2020) EP07 Eosinophilic granulomatosis with polyangiitis: diagnostic and therapeutic challenges during COVID-19 pandemic. Rheumatol Adv Pract. https://doi.org/10.1093/rap/rkaa052.006

13. Hashizume H, Sano Y, Furukawa S, Imokawa S (2020) Eosinophilic granulomatosis with polyangiitis mimicking coronavirus disease 2019: a case report. J Eur Acad Dermatol Venereol 34(10):e557-e559

14. Duran E, Kilic L, Durhan G et al (2020) Vital corner of diagnostic challenge: eosinophilic granulomatosis with polyangiitis or COVID-19 pneumonia? Ann Rheum Dis. https://doi.org/10.1136/ annrheumdis-2020-218533

15. Xie G, Ding F, Han L, Yin D, Lu H, Zhang M (2020) The role of peripheral blood eosinophil counts in COVID-19 patients. Allergy 76(2):471-482

16. Zhang J-J, Dong X, Cao Y-Y et al (2020) Clinical characteristics of 140 patients infected with SARS-CoV-2 in Wuhan. China Allergy 75(7):1730-1741

17. Du Y, Tu L, Zhu P et al (2020) Clinical features of 85 fatal cases of COVID-19 from Wuhan. A retrospective observational study. Am J Respir Crit Care Med 201(11):1372-1379

18. Li Q, Ding X, Xia G et al (2020) Eosinopenia and elevated C-reactive protein facilitate triage of COVID-19 patients in fever clinic: a retrospective case-control study. EClinicalMedicine 23:100375

19. Katzenstein A-LA (2000) Diagnostic features and differential diagnosis of Churg-Strauss syndrome in the lung: a review. Am J Clin Pathol 114(5):767-772

20. Bottero P, Bonini M, Vecchio F et al (2007) The common allergens in the Churg-Strauss syndrome. Allergy 62(11):1288-1294

21. Sinico RA, Di Toma L, Maggiore U et al (2005) Prevalence and clinical significance of antineutrophil cytoplasmic antibodies in Churg-Strauss syndrome. Arthritis Rheum 52(9):2926-2935

22. Sablé-Fourtassou R, Cohen P, Mahr A et al (2005) Antineutrophil cytoplasmic antibodies and the Churg-Strauss syndrome. Ann Intern Med 143(9):632-638

23. Conron M, Beynon HL (2000) Churg-Strauss syndrome. Thorax 55(10):870-877
24. Vaglio A, Moosig F, Zwerina J (2012) Churg-Strauss syndrome: update on pathophysiology and treatment. Curr Opin Rheumatol 24(1):24-30

25. Schramm MA, Venhoff N, Wagner D et al (2020) COVID-19 in a severely immunosuppressed patient with life-threatening eosinophilic granulomatosis with polyangiitis. Front Immunol. https:// doi.org/10.3389/fimmu.2020.02086

26. Du Vignaux CM, Ahmad K, Tantot J, Rouach B, Traclet J, Cottin V (2021) Evolution from hypereosinophilic bronchiolitis to eosinophilic granulomatosis with polyangiitis following COVID19: a case report. Clin Exp Rheumatol 39(1):11-12

27. Strangfeld A, Schäfer M, Gianfrancesco MA et al (2021) Factors associated with COVID-19-related death in people with rheumatic diseases: results from the COVID-19 global rheumatology alliance physician-reported registry. Ann Rheum Dis. https://doi.org/ 10.1136/annrheumdis-2021-220134

28. Huang C, Wang Y, Li X et al (2020) Clinical features of patients infected with 2019 novel coronavirus in Wuhan China. Lancet 395(10223):497-506

29. Yang X, Yu Y, Xu J et al (2020) Clinical course and outcomes of critically ill patients with SARS-CoV-2 pneumonia in Wuhan, China: a single-centered, retrospective, observational study. Lancet Respir Med 8(5):475-481

30. Tan L, Wang Q, Zhang D et al (2020) Lymphopenia predicts disease severity of COVID-19: a descriptive and predictive study. Signal Transduct Target Ther 5(1):1-3

31. Group RC (2020) Dexamethasone in hospitalized patients with Covid-19-preliminary report. N Engl J Med. https://doi.org/10. 1056/NEJMoa2021436

32. Mehta P, Porter JC, Chambers RC, Isenberg DA, Reddy V (2020) B-cell depletion with rituximab in the COVID-19 pandemic: where do we stand? Lancet Rheumatol 2(10):e589-e590

33. Florence A, Nassim AA, Jean-David A et al (2020) Severity of COVID-19 and survival in patients with rheumatic and inflammatory diseases: data from the French RMD COVID-19 cohort of 694 patients. Ann Rheum Dis. https://doi.org/10.1136/annrh eumdis-2020-218310

Publisher's Note Springer Nature remains neutral with regard to jurisdictional claims in published maps and institutional affiliations. 